\title{
Experimental Study with Enhanced Vision System Prototype Unit
}

\author{
VPS Naidu, Narayana Rao P., Sudesh K Kashyap, Shanthakumar N. and Girija G. \\ Multi Sensor Data Fusion Lab \\ Flight Mechanics and Control Division \\ CSIR - National Aerospace Laboratories \\ Bangalore-17, India \\ vpsnaidu@gmail.com
}

\begin{abstract}
The National Civil Aircraft (NCA) being developed at National Aerospace Laboratories (NAL) is expected to have the capability of operation from airports with minimal infrastructure and instrumentation facility under all-weather conditions. The key enabling technology for this is an Integrated Enhanced and Synthetic Vision System (IESVS), which is a combination of Enhanced Vision System (EVS), Synthetic Vision System (SVS), and Head-Up Display. A prototype of EVS consisting of Forward Looking Infrared (FLIR) camera and CCD color camera is developed and tested at NAL. A Simulink block is developed to acquire the image data in real time (online) from a four channel frame grabber. An image fusion algorithm based on wavelets is developed to fuse the images from CCD and FLIR cameras. The affine transform used for image registration is computed by selecting the control points from both CCD and FLIR images. Test results from the experiments conducted on the runway during day and night (runway lights ON/OFF) conditions are presented.
\end{abstract}

Keywords-National Civil Aircraft, Enhanced Vision System, ImageFusion, FLIR

\section{INTRODUCTION}

Increasing demand for air transportation puts the airline operators under tremendous pressure to maintain the timings of arrival and departure with no significant delay. The delay in flight operations depends upon various factors. One of the main factors that affect the flight operations is weather along the flight route. Reduced visibility due to adverse weather conditions makes it difficult to conduct flight operations in the same manner and rate as in visual meteorological conditions. Although navigation aids like Automatic Direction Finder (ADF), Distance Measuring Equipment (DME), VHF omnirange (VOR), Instrument Landing System (ILS), Microwave Landing System (MLS), Global Positioning System (GPS), etc. can provides solutions to many of the problems caused by low visibility, the potential now exists for providing enhanced visual references to the flight crew. Currently, several organizations around the world are working on Enhanced Flight Vision Systems (EFVS) and Synthetic Vision Systems (SVS) to provide solutions for this problem. According to FAA, EFVS is defined as a combination of Enhanced Vision System (EVS) and Head-Up Display (HUD). An Enhanced Vision System (EVS) is an electronic means to provide the flight crew with a sensor-derived or enhanced image of the external scene through the use of imaging sensors such as forward looking infrared, millimeter wave radiometry, millimeter wave radar, and/or low light level image intensifying. A Synthetic Vision System (SVS) is an electronic means to display a computer-generated image of the applicable external topography from the perspective of the flight deck that is derived from aircraft attitude, altitude, position, and a coordinate-referenced database.

Of late the NASA Langley Research Center (LaRC) Aviation Safety and Security Program Office and Systems Engineering Directorate have been initiating projects to improve air travel safety [1]. One of the initiating projects is the Synthetic Vision Systems (SVS) where innovative techniques to eliminate aircraft accidents caused due to poor visibility especially during landing and takeoff are being investigated. An Enhanced Vision System (EVS) combined with SVS is used to provide real-time scene from a vision sensor suite with Infra Red and visible cameras to a pilot. The vision sensors can provide more information about the environment than pilot observation during poor visibility conditions viz., fog, rain, snow or haze $[1,2]$. The main function of the EVS is to fuse the imaging sensor data to increase the information content and quality of the captured imagery, and to present the fused image instead of individual images to the pilot on head up display to increase the pilot situation awareness.

The National Civil Aircraft (NCA) proposed to be developed at National Aerospace Laboratories (NAL) is expected to have the capability of operation from airports with minimal infrastructure and instrumentation facility under all-weather conditions [3]. One of the technologies that hold promise for achieving this is the Integrated Enhanced and Synthetic Vision System (IESVS). IESVS can create near all-weather access to any touchdown zone at any landing facility while avoiding 
costs for land acquisition, approach lighting, ground-based precision guidance systems (ILS), radar and control tower infrastructure. The ability to see in all directions, even in reduced visibility conditions, offers considerable benefits for operational effectiveness and safety.

Multi Sensor Data Fusion (MSDF) group of Flight Mechanics and Control Division (FMCD)/NAL has embarked on the IESVS development for NCA. In the absence of real field data or hands on experience with EVS sensors, NAL has built a scale down EVS hardware prototype unit in association with $\mathrm{m} / \mathrm{s}$ Serial Innovation, India Pvt. Ltd. The EVS prototype unit consists of two cameras, one forward looking infrared (long wave infrared, LWIR with 7 to $12 \mu \mathrm{m}$ wavelength) and the other one electro-optical camera. This unit is field tested on a ground vehicle at HAL airport runway for generating data for studying issues related to sensor latency, FOV (Field of View) and design eye point location in addition to generating data for evaluation of sensor fusion algorithms. EVS developed as a part of IESVS is presented in this paper.

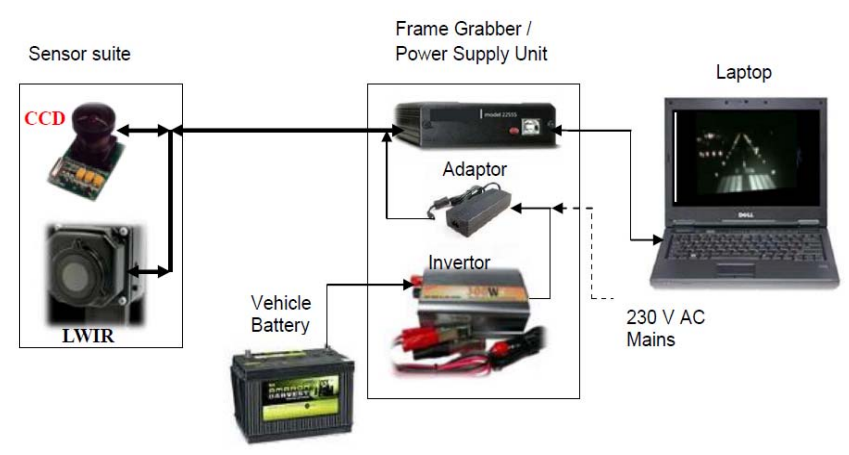

Fig-1. Connection diagram of prototype EVS

\section{EVS PROTOTYPE}

The information flow diagram of the EVS prototype is shown in Fig-1. The sensor suite consists of image sensing devices viz., Charge Coupled Device (CCD) camera and Long Wave InfraRed (LWIR) camera [4] are powered by $12 \mathrm{~V}$ DC from vehicles battery. CCD that detects $0.4-0.78 \mu \mathrm{m}$ band and it can capture runway markings, skyline, and city lights in good visibility conditions. LWIR senses radiation in $7.5-14 \mu \mathrm{m}$ band and it can capture background scenery, terrain features and obstacles at night and in other low visibility conditions. Vehicles battery of $12 \mathrm{~V}$ DC is fed to inverter where the inverter converts $12 \mathrm{~V} \mathrm{DC}$ to $230 \mathrm{~V} \mathrm{AC}$ and in-turn fed to $12 \mathrm{~V}$ DC adapter as shown in Fig-1. This arrangement is to avoid variation in the power supply if directly fed from the vehicle battery. For laboratory experiments, both battery and inverter can be bypassed by connecting $230 \mathrm{~V}$ AC mains directly to the $12 \mathrm{~V} \mathrm{DC}$ adapter. The image/video data gathered by the image sensing devices is fed to frame gabber. The frame grabber unit has four analog input channels that can acquire the data and output the digital frame data at a rate of $15 \mathrm{~Hz}$ by each channel
[5]. Since the prototype has two sensing devices only two channels will be active and the frame gabber could able to provide the image frames at a rate of $30 \mathrm{~Hz}$ by each of the two channels. The output of the frame gabber is fed to laptop for video acquiring and other image processing through the USB port. The frame grabber is powered by USB port of the laptop/PC.

The individual components/devices used in this experiment are shown in Fig-2. The integration of these devices is done at the laboratory and tested.

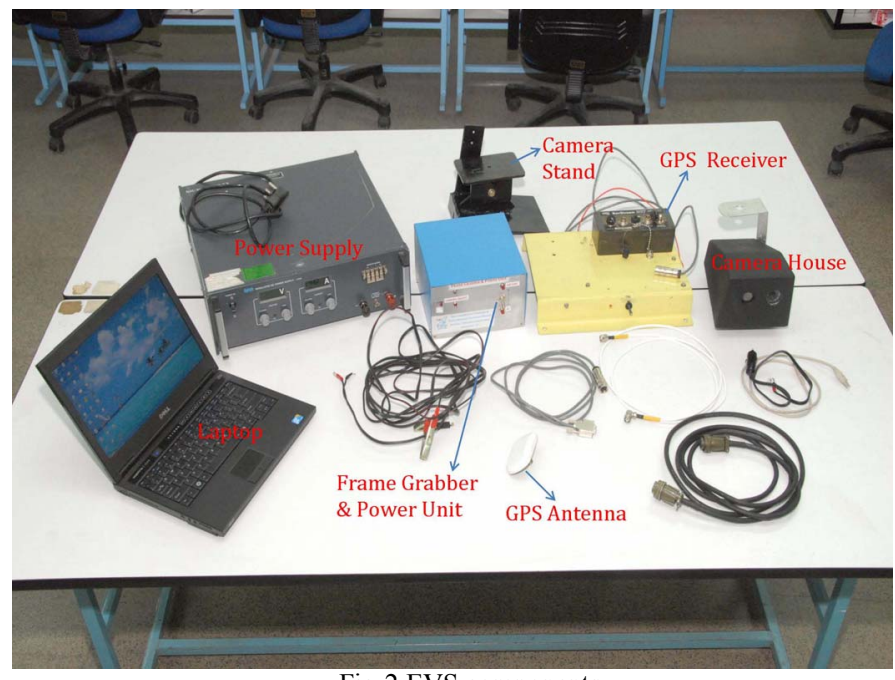

Fig-2 EVS components

\section{A. Processing}

The Matlab Simulink model of EVS system is shown in Fig-3. Image registration has been done since the videos are not bore sighted. The registered images are fused using wavelets.

\section{B. Image Acquisition}

Sensoray frame grabber (S2255) is used to acquire video data from CCD also known as EO (elctro optic) and LWIR imaging sensors in the development of Enhanced Vision prototype. Source code in $\mathrm{C}++$ is available in Sensoray website for interfacing the frame grabber to PC. The equivalent Matlab or Simulink code is not available. An attempt is made to develop the interface $\mathrm{C}++$ code in Simulink using legacy code [6]. The developed Simulink block is shown in Fig-4.

\section{Image Registration}

The acquired data is converted to double precision since the image registration and image fusion algorithms work on data in double format. Unregistered images from CCD and LWIR cameras taken at MSDF Lab are shown in Fig 5 (top). Image registration [7-9] is done by selecting the control points from EO and LWIR images of size 640 x 480. The selected control points are: 
EO points $=[584$ 146; 306 204; $113419 ; 178$ 161; 535 367; $431368 ; 171411 ; 14309]$;

LWIR points $=[622$ 135; 357 196; 172 438; 238 148; 577 384; 485 386; 232 432; 84 313];

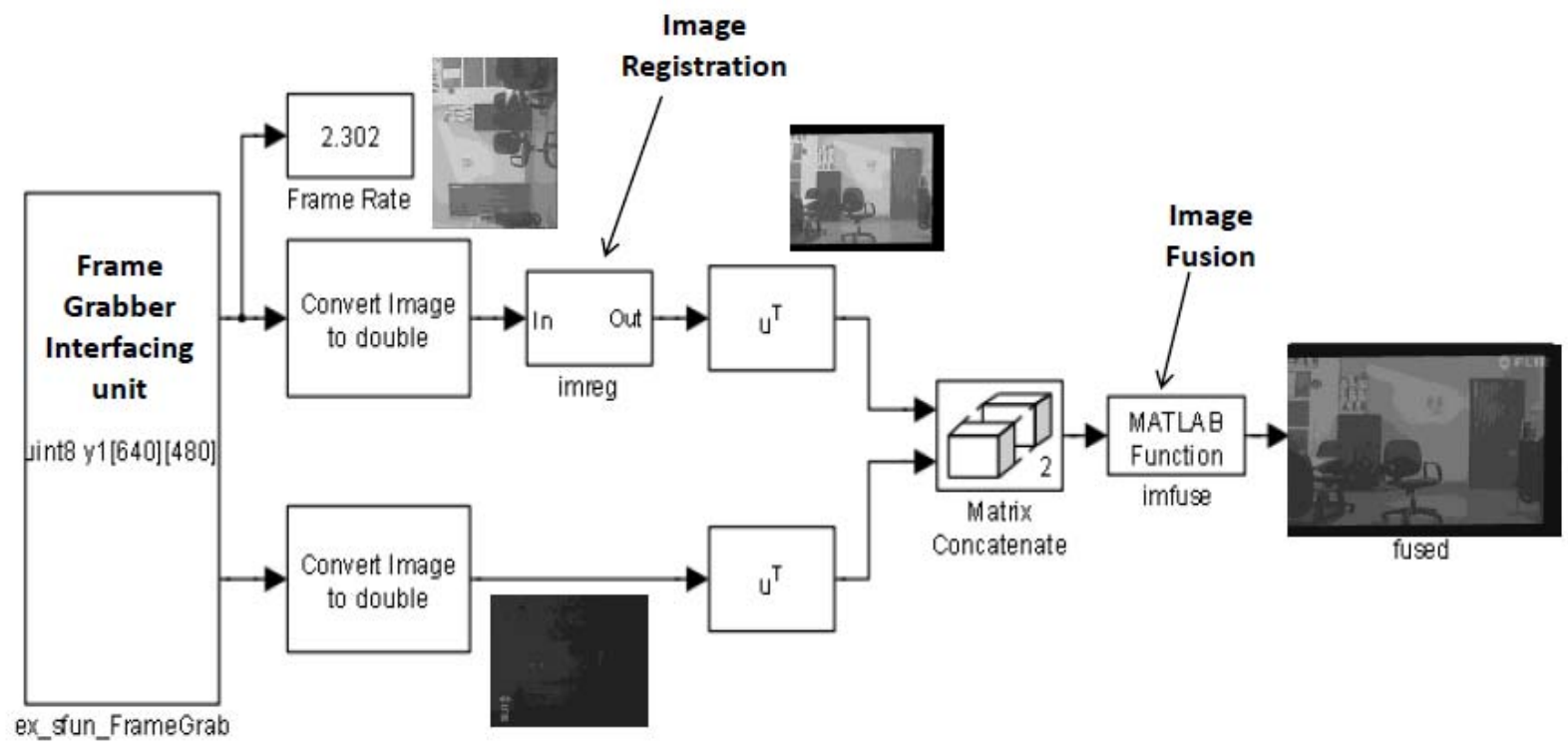

Fig-3 Simulink model of EVS prototype

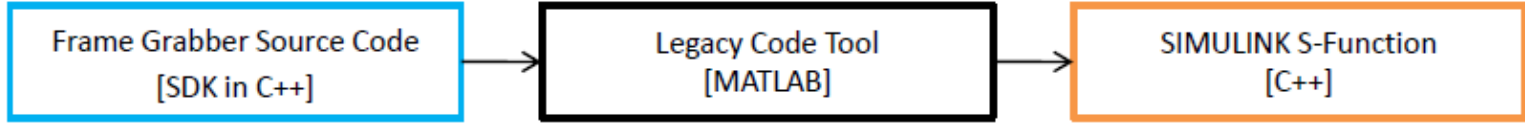

Fig-4 Legacy code for frame grabber interfacing unit

The following matlab function is used to find the affine transform between the two images.

tmatrix $=$ cp2tform(EO_points,LWIR_points,'affine');

The tmatrix is a structure as:

ndims_in: 2

ndims_out: 2

forward_fcn:@fwd affine

inverse_fcn:@inv_affine

tdata: [1x1 struct]

The affine transform is:

$$
\text { tmatrix }=\text { t.tdata } . T=\left[\begin{array}{ccc}
1.0356 & -0.0092 & 0 \\
0.0270 & 0.8817 & 0 \\
-52.4870 & 32.9069 & 1
\end{array}\right]
$$

The simulink block for image registration is shown in Fig-6 and registered image obtained from this block is shown in Fig5 (bottom). Since the cameras are fixed, the affine transform obtained earlier can be used for subsequent experiments.

\section{Image Fusion}

The registered images are fused using wavelets with one level of decomposition [10,11]. The complete level decompositions may be represented as: 


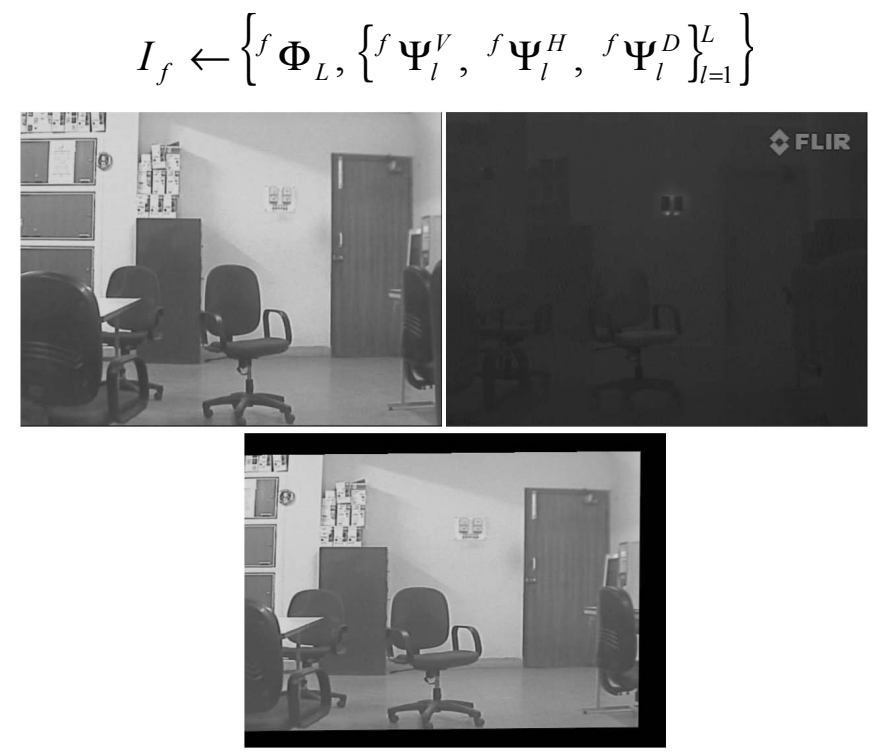

Fig-5 Unregistered (top) and registered (bottom) images

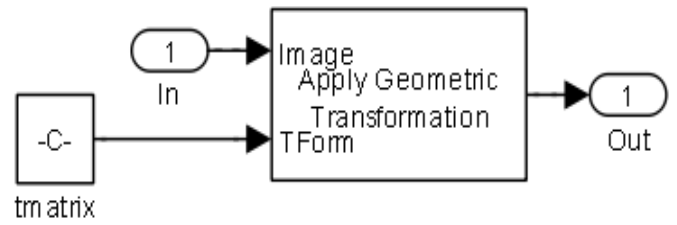

Fig-6 Simulink block for image registration

\section{EXPERIMENTAL RESULTS}

After laboratory experiments the EVS prototype unit along with GPS receiver is mounted on the ground test vehicle to carry out tests on the airport runway. The closed view of the sensor suit along with GPS antenna mounted on the test vehicle is shown in Fig 8. Frame grabber \& power supply unit

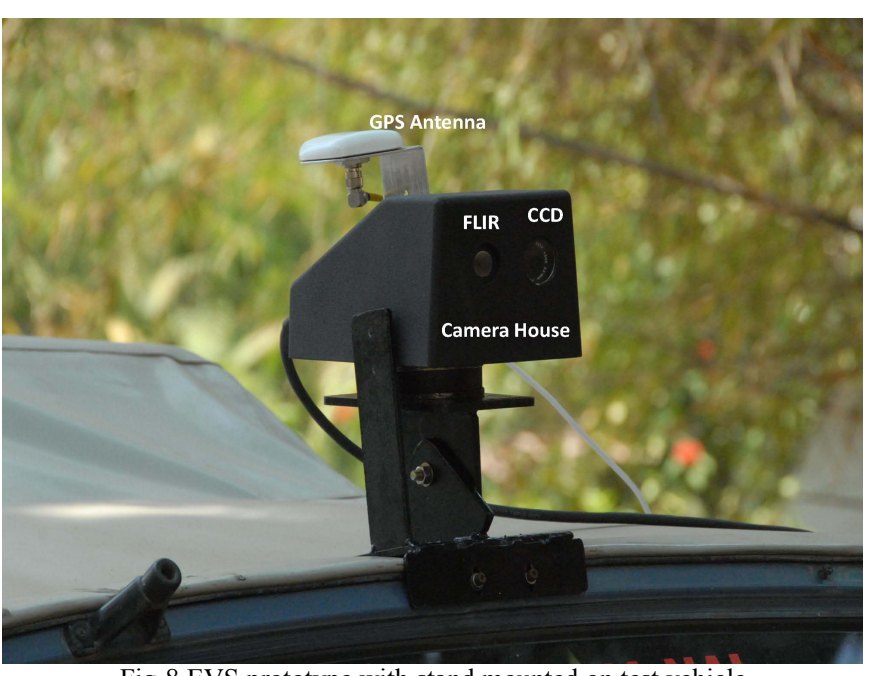

Fig-8 EVS prototype with stand mounted on test vehicle

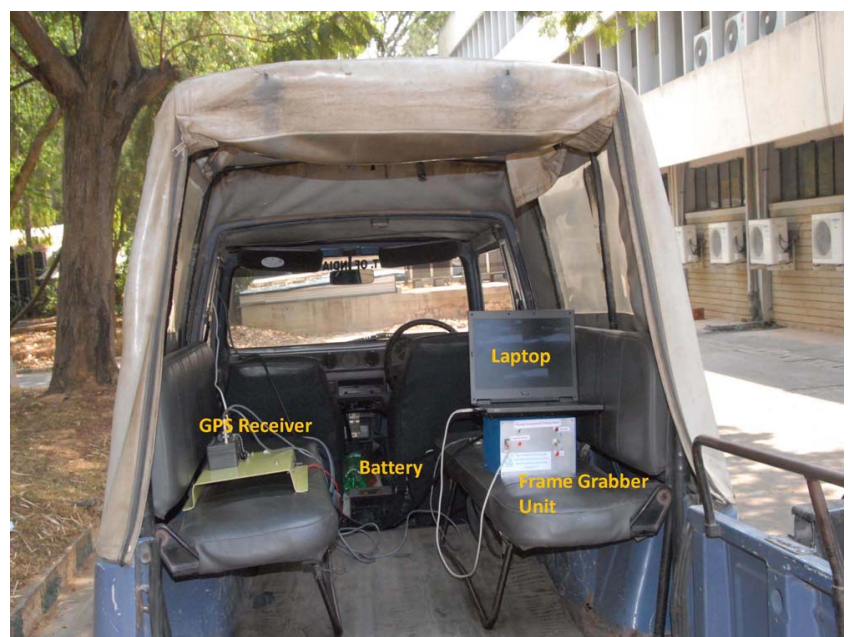

Fig-9 Rear view of the test vehicle

Experiments were conducted on runway on 11th Jan 2010 during day time and 13th Jan 2010 after sunset. During both

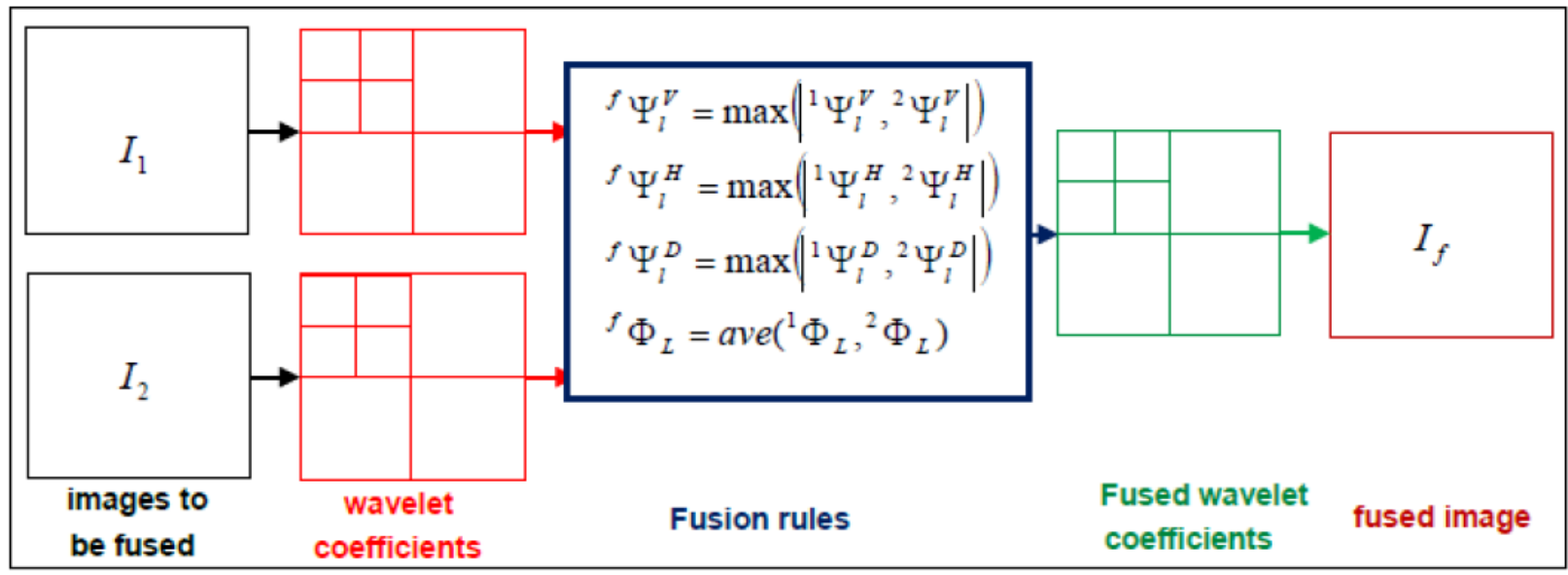

Fig-7 Schematic diagram of the wavelet image fusion scheme

and laptop are placed inside the vehicle as shown in Fig-9 for recording the data on line. the days test vehicle was driven on the run way from one end to the other end and return. During the run, video was captured from both the cameras, fused online and recorded on the 
laptop. Fig-10 shows the image recorded by CCD camera and LWIR (FLIR Forward looking Infrared) camera on the runway after sunset with runway lights ON. From the figure it can be seen that only runway lights are visible in CCD camera and not the other features of the runway, whereas in LWIR camera it is the other way i.e. only runway markings are visible. Once both the images are fused with proper image registration (ref from section IIc), the fused image contains all the necessary information of the runway and hence pilot can have better situational awareness of the runway.

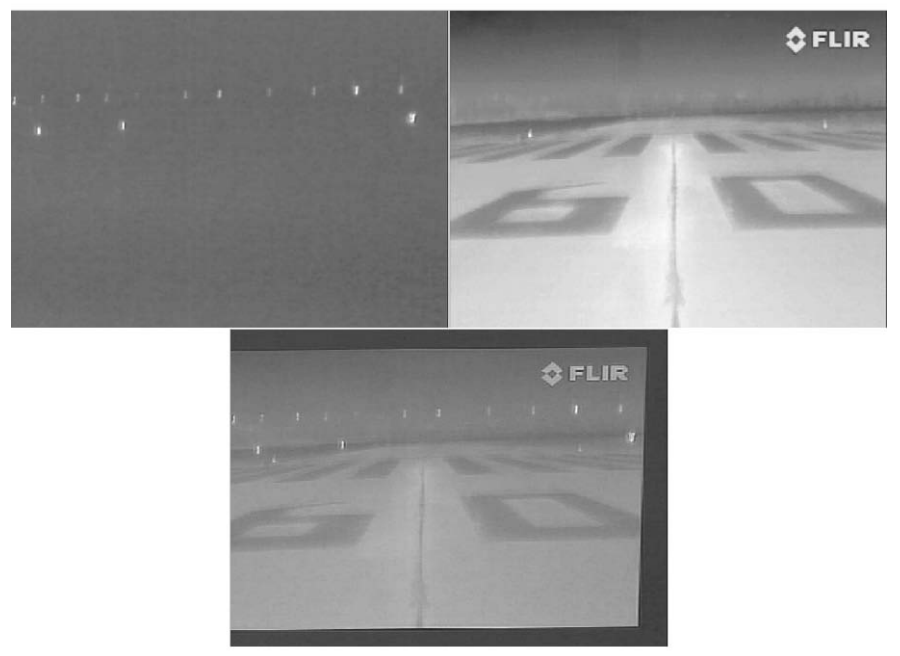

Fig-10 Runway view

Objective of this experiment is to study the characteristics of the image sensors across different weather conditions during day and night. The data generated will also be useful to evaluate the image fusion algorithms developed in-house for automatic target recognition (ATR) research. Apart from images of aircraft and helicopters landing, taxing and takeoff, images containing runway markers were also acquired for the purpose of runway feature extraction. It is also proposed to utilize this data to arrive at fusion quality metrics based on the visibility criterion.

\section{CONCLUSION}

Enhanced vision system (EVS) prototype is developed and tested as part of integrated enhanced synthetic vision system (IESVS). Legacy code for frame grabber interfacing unit has been developed to grab the video in Simulink environment to test the EVS prototype in real time. Affine transform is computed by choosing the control points from CCD and LWIR images for image registration and it can be used for subsequent experiments since the imaging sensors are fixed in sensor suite. Wavelet based image fusion algorithm has been used to fuse the CCD and LWIR images. EVS prototype is tested at airport during day and night (with runway lights $\mathrm{ON}$ and OFF) conditions. The fused image contains all the information/features of runway and hence pilot can have better situational awareness of the runway. The knowledge gained from this prototype experiment would be very useful for integrating the synthetic vision and also for automatic target recognition of runway obstacles.

\section{REFERENCES}

[1] Glenn D. Hines, Zia-ur Rahman, Daniel J. Jobson, Glenn A. Woodell and Steven D. Harrah, "Real-time enhanced vision system", Enhanced and synthetic vision 2005, Edited by verly, Jacques G. Proceedings of the SPIE, Vol. 5802, pp.127-134 (2005).

[2] Peter Hecker, Hans-Ullrich Doehler, Reiner suikat, "Enhanced vision meets pilot assistance", Enhanced and synthetic vision 1999, Edited by verly, Jacques G. Proceedings of the SPIE, Vol. 3691, pp.125-136 (1999).

[3] Girija Gopalratnam, "Data fusion for integrated enhanced vision system for transport aircraft", project proposal, FMCD, NAL, Dec. 2007.

[4] http://www.flir.com

[5] http://www.sensoray.com/products/2255.htm

[6] http://www.mathworks.de/access/helpdesk/help/toolbox/simulink/slref/le gacy_code.html

[7] http://www.mathworks.com/ access/helpdesk/help/toolbox/images/f2014983.html

[8] http://www.math.tau.ac.il/turkel/notes/registration.pdf

[9] Barbara Zitova and Jan Flusser, "Image registration metods: a survey", Image and vision computing, Vol.21, pp.977-1000, 2003.

[10] Gonzalo Pajares and Jesus Manuel de la Cruz, "A Wavelet-based Image Fusion Tutorial”, Pattern Recognition, Vol.37, pp.1855-1872, 2004.

[11] VPS Naidu and J.R. raol, "Pixel-Level Image Fusion using Wavelets and Principal Component Analysis: A Comparative Study", Defence Science Journal, Vol. 58, pp.338-352, May 2008. 